\title{
Dynamics of water nanodroplets and aqueous protons in non-ionic reverse micelles
}

\author{
J. Rodriguez, ${ }^{a b}$ D. Laria, ${ }^{a b}$ E. Guàrdia ${ }^{* c}$ and J. Martí ${ }^{* c}$ \\ Received 25th September 2008, Accepted 28th November 2008 \\ First published as an Advance Article on the web 21st January 2009 \\ DOI: $10.1039 / \mathbf{b 8 1 6 8 2 7 j}$
}

\begin{abstract}
We present a study of the microscopic dynamics of water trapped in reverse non-ionic micelles by means of a series of molecular dynamics simulations. The analysis of the effects of micellar confinement on spectroscopical properties of an excess proton has also been considered. Our micelles were microemulsions made with the neutral surfactant diethylene glycol monodecyl ether $\left[\mathrm{CH}_{3}\left(\mathrm{CH}_{2}\right)_{11}\left(\mathrm{OC}_{2} \mathrm{H}_{4}\right)_{2} \mathrm{OH}\right]$. Simulation experiments including the proton species were performed using a multistate empirical valence bond Hamiltonian model. Diffusion of water in the micelle is markedly slower than that in the bulk liquid, in the same fashion as happens with reorientational dynamics. Spectral densities of hydrogens revealed a blue-shift of the $\mathrm{OH}$-stretching vibration together with a split of the main band into two components. Absorption lineshapes of the solvated proton in the vicinity of the internal surface of the micelle indicate the coexistence of Eigen-like and Zundel-like structures and a tendency to red-shifting (compared to the aqueous unconstrained excess proton case) of the two relevant spectral bands (around 2000 and 2500 wavenumbers) mainly due to the slower dynamics of proton vibrations in water near interfaces.
\end{abstract}

\section{Introduction}

Reverse micelles are quasi-spherical aggregates of surfactants composed of hydrophilic headgroups, eventually including ionic or nonpolar species and hydrocarbon based tailgroups, with hydrophobic characteristics ${ }^{1-3}$ immersed in non-polar macroscopic phases. The addition of polar solvents such as water leads to the formation of water nanodroplets in the internal core of the micelle. These water nanopools are strongly influenced by the presence of the interface which produces important changes in the structure and dynamics, when compared to those of the bulk liquid. ${ }^{4}$ Reverse micelles are often described by the ratio $w_{0} \equiv\left[\mathrm{H}_{2} \mathrm{O}\right] /[$ surfactant]. Usually experimental setups have employed large probe molecules able to characterize the interfacial region and to study solvation dynamics in the water pool, ${ }^{5}$ although recent works have been focussed directly on the water properties by means of ultrafast infrared (IR) spectroscopy. ${ }^{6-8}$

In most of the reported studies of aqueous reverse micelles, at least two classes of water molecules are considered: (i) those located nearby surfactant groups and, eventually, counter-ions, which are particularly affected by confinement effects and (ii) water molecules residing in the internal part of the micellar pool, which receive a milder influence of the micellar interface. It has been observed that water structure exhibits important modifications compared to that of the bulk

${ }^{a}$ Departamento de Química Inorgánica, Analítica y Química-Física, e INQUIMAE, Facultad de Ciencias Exactas y Naturales, Universidad de Buenos Aires, Ciudad Universitaria, Pabellón II, 1428, Argentina

${ }^{b}$ Unidad Actividad Química, Comisión Nacional de Energía Atómica, Avenida Libertador 8250, 1429 Buenos Aires, Argentina.

E-mail: javier@speedy.cnea.gov.ar;dhlaria@cnea.gov.ar

${ }^{c}$ Departament de Fisica i Enginyeria Nuclear, Universitat Politècnica de Catalunya, B4-B5 Campus Nord, 08034 Barcelona, Catalonia, Spain.E-mail: elvira.guardia@upc.edu,jordi.marti@upc.edu liquid - more notably for interfacial molecules and for small micellar sizes - whereas water dynamics is much less affected. Nevertheless, even more relevant than size is the location of water molecules in the nanodroplet: in particular, interfacial water molecules exhibit different spectral IR absorption than those pertaining to the internal part of the pool. ${ }^{7}$ Keeping these issues in mind, we have focussed our efforts in the detailed analysis of spectral features for pure water as well as for protonated aqueous samples for a nonionic micelle (diethylene glycol monodecyl ether) of small size $\left(w_{0} \sim 2.5\right)$. In a previous work, structural aspects of the solvation of protons in such micellar environments were considered. ${ }^{9}$ In this work, dynamical aspects such as rotational and translation diffusion and the spectroscopy of water in the micelle are addressed. Furthermore, for the first time, we also examined the spectral characteristics of an excess proton embedded in an inner pool in an aqueous reverse micelle.

This article is organized as follows: first we will briefly describe the models and methods employed in the simulations. Second, the results on diffusion, reorientation and spectroscopy of pure water in the micellar pool are discussed. Third, spectral densities of the excess proton are analyzed and, finally, several important conclusions are presented.

\section{Models and computational details}

Two series of molecular dynamics (MD) simulations have been carried out: one for pure water inside a non-ionic reverse micelle composed by the neutral surfactant diethylene glycol monodecyl ether $\left[\mathrm{CH}_{3}\left(\mathrm{CH}_{2}\right)_{11}\left(\mathrm{OC}_{2} \mathrm{H}_{4}\right)_{2} \mathrm{OH}\right]$, referred from here on as $\mathrm{C}_{12} \mathrm{E}_{2}$, and a second one including an excess proton dissolved in the inner pool of the micelle. A snapshot of the latter system is presented in Fig. 1. 


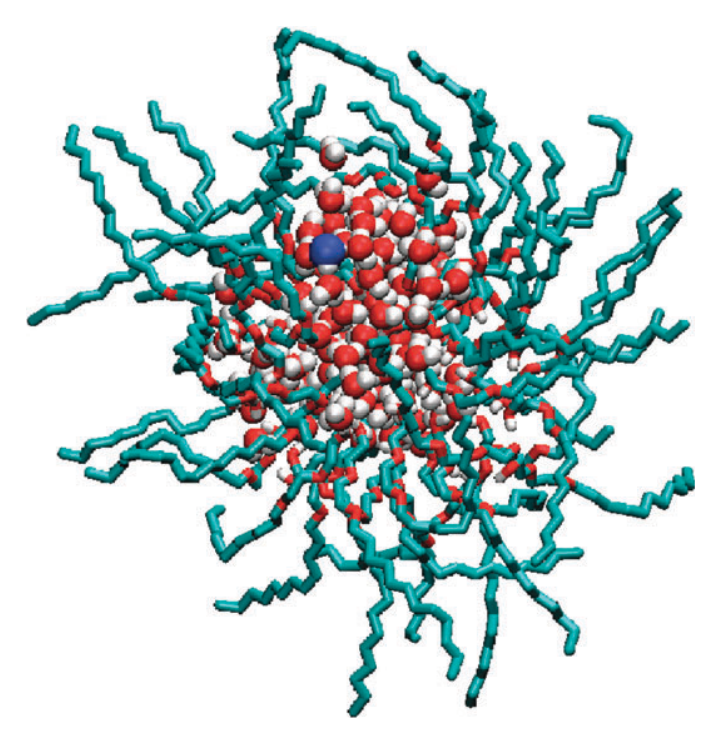

Fig. 1 Snapshot of the protonated non-ionic aqueous $\mathrm{C}_{12} \mathrm{E}_{2}$ micelle in equilibrium at room temperature. Oxygens (red), hydrogens (white), surfactants (cyan), hydronium (blue, larger size).

In order to construct the $\mathrm{C}_{12} \mathrm{E}_{2}$ reverse micelle, we followed a procedure introduced by Allen et al. ${ }^{10}$ This procedure is briefly explained in ref. 9 where we refer the interested reader for details. The inner pool contained $N_{w}=119$ water molecules surrounded by $N_{s}=50$ surfactant molecules at $T=298 \mathrm{~K}$. Since the incorporation of an external non-polar phase has been shown to introduce only minor effects on the microscopic properties of the water nanodroplet, ${ }^{10}$ all simulation experiments were carried out in vacuo.

Water molecules were classified into two groups: the first one named surface water corresponds to molecules located in the external part of the inner pool, while the second one included the rest of the bulk-like water. The criterion employed to perform this classification was based on a plain geometric argument: a water molecule was considered as belonging to the interfacial shell if its center of mass was located at a radial distance $r \leq 3 \AA$ from any of the surfactant sites (see ref. 9). By applying this criterion, $\sim 63 \%$ of the water molecules were found to be interfacial while the remaining $\sim 37 \%$ correspond to "bulk-like" water. In passing, we should remark that the implementation of more sophisticated selection rules, such as distinguishing water molecules doubly-hydrogen-bonded to oxygen sites in the surfactant, ${ }^{11}$ produced no significant differences in the results that will follow. We think that for a small, hydrophilic micelle such as $\mathrm{C}_{12} \mathrm{E}_{2}$, dynamics of water forming four hydrogen-bonds is not significantly different to that of water in bulk, although some changes at the quantitative level especially concerning rotational dynamics and diffusivity have been observed, as will be shown below. Conversely, in ionic micelles such as in phosphate fluorosurfactant $^{12}$ the definition of several regions has produced dramatic changes in dynamics, such as in translational diffusion.

The excess proton has been modeled by means of an empirical valence bond (EVB) model. Details of this methodology have been extensively described in previous works. The particular water and hydronium models employed in this work have been adapted from those reported by Voth and co-workers. ${ }^{13-17}$ Some of the main details are as follows: dynamics of the system was obtained from the ground state of an EVB Hamiltonian, $\hat{H}_{\mathrm{EVB}}$, whose matrix elements are functions exclusively of the nuclear coordinates $\{\mathbf{R}\}$. At each step of the simulation, the instantaneous diabatic states (those describing the localization of the excess proton in a tagged water molecule) are determined, from a connectivity pattern established by sequences of hydrogen bonds. The index of the largest coefficient of the expansion of ground state, $c_{i}^{2}$, determines the instantaneous location of the proton, which can eventually be updated, in the advent of a proton translocation episode. Diagonal elements $h_{i i}(\{\mathbf{R}\})$ included inter- and intramolecular interactions with the tagged $\mathrm{H}_{3} \mathrm{O}^{+}$group and the rest of the water molecules, that were modeled using a flexible version of the TIP3P model. ${ }^{15}$ In addition, these interactions were supplemented with contributions involving surfactant sites. Using the united atom limit for the $\mathrm{CH}_{3}$ and $\mathrm{CH}_{2}$ groups, $\mathrm{C}_{12} \mathrm{E}_{2}$ molecules $\left[\mathrm{CH}_{3}\left(\mathrm{CH}_{2}\right)_{11}\left(\mathrm{OC}_{2} \mathrm{H}_{4}\right)_{2} \mathrm{OH}\right]$ comprised a total of 20 interaction sites. Intra-molecular interactions in the surfactant molecules included stretching, bending, non-bonding and dihedral modes; energy, length and charge parameters for these interactions were taken from refs. 18-20. Statistical dynamical properties were obtained from simulation runs lasting $5 \mathrm{~ns}$. In all cases, the Ewald summation method has been used in order to account for long-range Coulombic forces.

\section{Results}

\subsection{Reorientational motions and diffusive behavior of pure water}

In MD simulations, reorientational motions of water are usually analyzed by examining specific time correlation functions $C_{l, \alpha}(t)$ defined by:

$$
C_{l, \alpha}(t) \equiv\left\langle P_{l}\left(\vec{u}_{\alpha}(t) \cdot \vec{u}_{\alpha}(0)\right)\right\rangle,(l=1,2)
$$

where $P_{l}$ is the $l$-th Legendre polynomial and $\vec{u}_{\alpha}$ represents a unit vector along a given molecular direction. We employed two unit vectors: $\vec{u}_{\mu}$, which coincides with the direction of the instantaneous molecular dipole moment and $\vec{u}_{H H}$ which is aligned along the instantaneous $\mathrm{H}-\mathrm{H}$ intramolecular direction. The two $C_{l, \alpha}(t)$ provide estimates for time scales associated with different dynamical modes which can be recorded from direct experimental measurements: more specifically, $C_{1, \mu}(t)$ is related to dielectric relaxation measurements ${ }^{21}$ and $C_{2, \mathrm{HH}}(t)$ can be inferred from ${ }^{1} \mathrm{H}-{ }^{1} \mathrm{H}$ dipolar relaxation NMR experiments. ${ }^{22}$

Results for correlation functions $C_{l, \mu}$ and $C_{l, \mathrm{HH}}$ are depicted in Fig. 2. At first glance, one can see that the decays of $C_{1, \alpha}$ are slower than those of $C_{2, \alpha}$ for the dipole moment and $\mathrm{HH}$ directions. The so-called molecular reorientational times $\tau_{1, \alpha}, \tau_{2, \alpha}$ along each direction can be obtained from the analysis of the behavior of $C_{l, \alpha}(t)$ after a first inertial transient. In all cases, we found that in this temporal regime, i.e. $t \sim 1 \mathrm{ps}$, the curves can be reasonably well described by single exponentials, whose decays $\tau$ are listed in Table 1. For the sake of comparison, $\tau$ obtained from a flexible TIP3P model for bulk water at ambient conditions is reported as well. We can observe that 


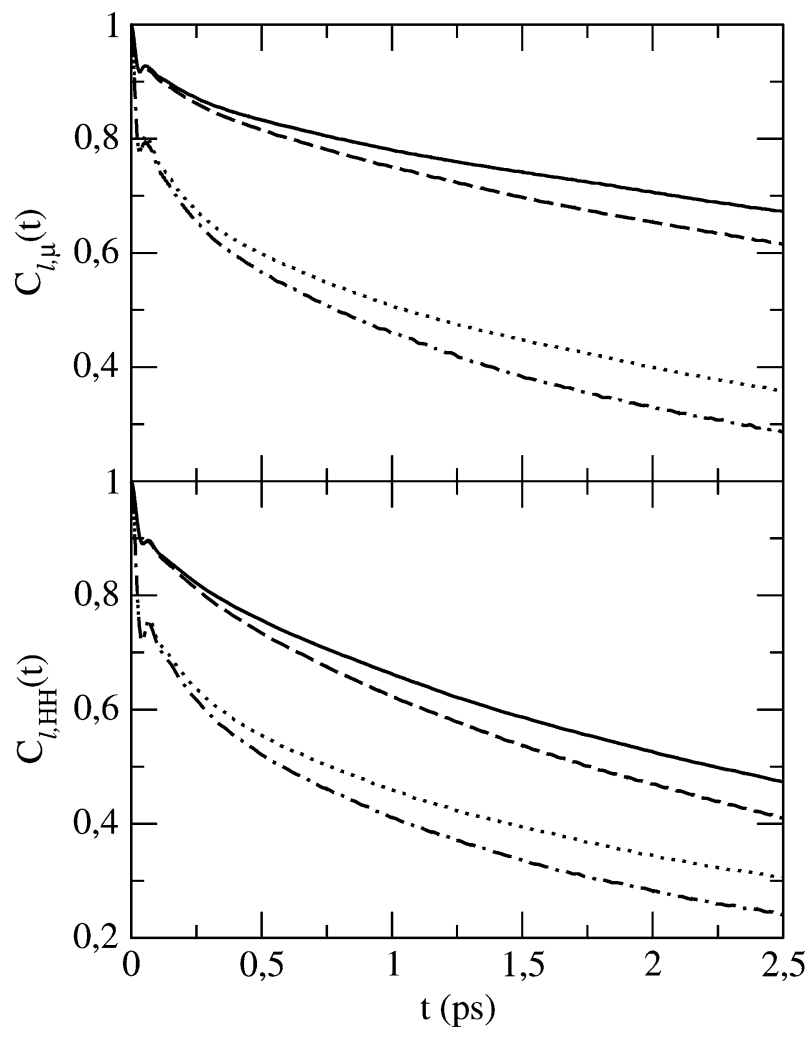

Fig. 2 Dipole moment $\left(C_{l, \mu}(t)\right)$ and HH-direction $\left(C_{l, H H}(t)\right)$ reorientational correlation functions. $C_{1, \alpha}$ for water at the interface (full line), $C_{1, \alpha}$ for bulk-like water (dashed line), $C_{2, \alpha}$ for water at the interface (dotted line), $C_{2, \alpha}$ for bulk-like water (dot-dashed line).

Table 1 Reorientational times of water $\tau_{1}$ and $\tau_{2}$ for dipole moment and $\mathrm{HH}$ directions (given in ps) for water located in the two selected regions. Estimated precision is about 0.1 ps in all cases. Oxygen selfdiffusion coefficients $D$ are given in $10^{-5} \mathrm{~cm}^{2} / \mathrm{s}$, with an estimated precision of $0.1 \times 10^{-5} \mathrm{~cm}^{2} / \mathrm{s}$. Additional simulation data available are also reported

\begin{tabular}{lrllll}
\hline Region & $\tau_{1, \mu}$ & $\tau_{2, \mu}$ & $\tau_{1, H H}$ & $\tau_{2, H H}$ & $\mathrm{D}$ \\
\hline Interfacial water & 10.2 & 4.4 & 4.5 & 3.7 & 2.5 \\
Bulk-like water & 7.6 & 3.2 & 3.6 & 2.8 & 2.9 \\
Flexible TIP3P $^{23}$ & 2.2 & 0.9 & 1.6 & 0.8 & 4.5 \\
\hline
\end{tabular}

$\tau$ values obtained from computer simulations of unconstrained bulk TIP3P water ${ }^{23}$ are markedly smaller than those reported here. This would confirm that the micellar confinement tends to slow down orientational correlations, in line with that suggested by the non-linear IR experiments of Piletic et $a l^{8,24}$ or by Dokter et al., ${ }^{7}$ who employed mid-infrared ultrafast pump-probe spectroscopy for several classes of reverse micelles. Moreover, note also that the previous observed trends are significantly enhanced as one singles out exclusively the contributions from interfacial water.

Concerning translational diffusion, we computed oxygen self-diffusion coefficients $D$ from time integrals of velocity autocorrelation functions, by discriminating water molecules located in the above mentioned regions. The results have been reported in Table 1. In a similar fashion to what we found for orientational dynamics, our simulated $D$ values in the center of the water pool (bulk-like) are smaller than the value of
$4.5 \times 10^{-5} \mathrm{~cm}^{2} / \mathrm{s}$ reported from simulations of unconstrained bulk water with a flexible TIP3P model. ${ }^{23}$ Similar results were reported by Allen et al. ${ }^{10}$ for $\mathrm{SPC} / \mathrm{E}$ water inside the $\mathrm{C}_{12} \mathrm{E}_{2}$ micelle. Concerning interfacial water, its diffusion is slightly smaller, $\sim 15 \%$. This observation is in good qualitative agreement with findings of Faeder and Ladanyi, ${ }^{25}$ who employed a somewhat simpler description of the reverse micelle than the one presented in this work. The overall picture that emerges from our dynamical results would suggest that the combined effects of spatial confinement and hydrogen-bonding intermolecular connectivity leads to remarkably stable water structures which are - eventually-much more stable than the usual tetrahedral structure which is characteristic of pure bulk water. Before closing this section, we would like to point out that our computer simulation results, like those previously reported by other authors such as Faeder and Ladanyi or Allen et al., lead to similar water diffusional trends to those indirectly inferred from experiments, ${ }^{26-29}$ i.e. overall retardations operated in all dynamical modes of water trapped in the cores of reverse micelles.

\subsection{High frequency spectroscopy: pure water and excess proton}

Important aspects pertaining to the solvation of excess protons embedded in the aqueous pool of a reversed micelle can be experimentally explored by means of spectroscopic measurements (infrared and Raman spectroscopy, timeresolved fluorescence). Infrared spectroscopy of water is known to be very sensitive to the local environment, especially to the characteristics of the architecture of its hydrogen-bond network. ${ }^{30}$ In order to unveil such structural characteristics in micelles, we examined two different species (water and the excess proton) in bulk and confined environments and computed appropriate time correlation functions that, in Fourier space, may reveal important features concerning interand intramolecular dynamics.

First, the infrared absorption lineshape $I(\omega)$ associated with the excess proton has been obtained from the Fourier transform of the time autocorrelation function of the time derivative of the dipole moment (see ref. 31) as follows:

$$
I(\omega) \propto \int_{0}^{\infty} e^{-i \omega t}\langle\dot{\vec{\mu}}(t) \dot{\vec{\mu}}(0)\rangle \mathrm{d} t
$$

Within the EVB framework, the time derivative of the dipole moment $\vec{\mu}(t)$ includes contributions from nuclear velocities and polarization fluctuations. ${ }^{17,32}$ To calculate $\dot{\vec{\mu}}(t)$, we considered exclusively the six instantaneous valence bond states exhibiting the largest weights $c_{i}^{2}$ and the estimates for the time derivatives of the different $c_{i}^{2}$ were obtained from standard first order perturbation theories in the form:

$$
\dot{c}_{i}=\sum_{n} \frac{\left\langle\Psi_{n}\left|\dot{\hat{H}}_{\mathrm{EVB}}\right| \Psi_{0}\right\rangle\left\langle\Phi_{i} \mid \Psi_{n}\right\rangle}{E_{0}-E_{n}}
$$

where $\left|\Psi_{n}\right\rangle$ represents the $n$-th eigenvector of $\hat{H}_{\mathrm{EVB}},\left|\Phi_{i}\right\rangle$ is the $i$-th vector of the diabatic basis set and $E_{n}$ is the $n$-th state eigenvalue of $\hat{H}_{\mathrm{EVB}}$. In addition, the vibrational spectral densities of water molecules $S_{H}(\omega)$ were obtained in the usual 
fashion, i.e. from the corresponding hydrogen velocity autocorrelation functions (see ref. 33).

Results for the spectra are shown in Fig. 3 (pure water) and 4 (excess proton). We use arbitrary units to present both, $I(\omega)$ and $S(\omega)$, since our calculations do not provide quantum information about line intensities. Given these restrictions, we will focus attention on spectral band shifts. Following a similar procedure as the one implemented to analyze the dynamics, the spectral densities of hydrogens lying inside the micellar pool (Fig. 3) have been separated into two contributions: bulk-like water molecules around the center of the pool and interfacial water nearby the internal surface of the reverse micelle. Spectral densities for unconstrained flexible TIP3P water are also included for comparison. No significant differences between the spectra of water from the two classes and spectra of bulk unconstrained water in the librational region $\left(300-800 \mathrm{~cm}^{-1}\right)$ are observed. We can conclude that this property is not sensitive to the subtle changes detected in the analysis of reorientational correlations presented above. In other words, molecular rotations along local molecular axis ${ }^{34}$ associated to this frequency region remain basically unaffected by the water confinement in the micellar core.

Conversely, the study of the $\mathrm{OH}$-stretching band-taking into account the broad maximum around $\omega_{\text {ref. }}=3650 \mathrm{~cm}^{-1}$ for bulk unconstrained flexible TIP3P water-reveals two main features for micellar water: (i) first, the split of the main band into two branches at both sides of the reference; (ii) second, spectral shifts for the two classes of water. Let us analyze these facts in deeper detail: the split of the $\mathrm{OH}$-stretch into two parts has been previously observed in other constrained water systems, such as water in restricted geometries (non-charged graphene slabs, ${ }^{35}$ carbon nanotubes ${ }^{36,37}$ ). This feature is normally attributed to hydrogen-bond breaking, which leads to a significant amount of free (non-bonded) hydrogens and to $\mathrm{OH}$-stretching frequencies closer to the gas phase values $^{38}\left(\sim 3800 \mathrm{~cm}^{-1}\right)$. The remaining water molecules, whose hydrogens are involved in intermolecular connectivity should be the ones that contribute to the lower frequency branch. Concerning spectral shifts, we remark that ultrafast infrared pump-probe spectroscopy experiments have revealed the existence of such frequency shifts in the case of ionic micelles such as in Aerosol-OT (AOT) ${ }^{8,39}$ or in the cetyltrimethylammonium bromide micelle (CTAB), ${ }^{7}$ which are usually explained in terms of the influence of confinement and ionic charges on the structure of the hydrogen-bond network. In our case, three different spectral shifts were observed: (i) $\Delta \omega \sim 30 \mathrm{~cm}^{-1}$ for both interfacial and bulk-like water; (ii) $\Delta \omega \sim-25 \mathrm{~cm}^{-1}$ for water at the interface and (iii) $\Delta \omega \sim-40 \mathrm{~cm}^{-1}$ for bulk-like water. Here, the magnitude of the spectral shifts has been defined as $\Delta \omega \equiv \omega-\omega_{\text {ref., }}$, leading to positive (negative) values for blue (red) shifts. Usually, $\mathrm{OH}$ vibrations of hydrogen-bonded water molecules located within micellar environments are red-shifted for both water classes and this should be interpreted as a direct consequence of the confinement. However, the reasons why the vibrational frequency of $\mathrm{OH}$ bonds of water in the central region is smaller than that of observed for interfacial water remain unclear. We could speculate that this feature would be indicative that, at the interface, the geometry and characteristics of hydrogen-bonding are somewhat different from those of bulk-like water, leading to looser structures, in line with that suggested by Dokter et al. ${ }^{40}$

Similar trends are observed in the absorption lineshape of the excess proton presented in the righthand plot of Fig. 4. The comparison between results obtained for bulk water protons and those from protons inside the micellar water pool indicate that stretching vibrations of the proton are red-shifted by $\Delta \omega \sim-20 \mathrm{~cm}^{-1}$. This is in good agreement with the results reported above (Fig. 3) and would indicate that confinement in the micellar environment generally leads to red-shifts in the $\mathrm{OH}$-stretch. In the lefthand plot of Fig. 4 we present a special feature of the excess proton spectrum, located in the region of $1800-2800 \mathrm{~cm}^{-1}$ and usually seen as a broad band with two
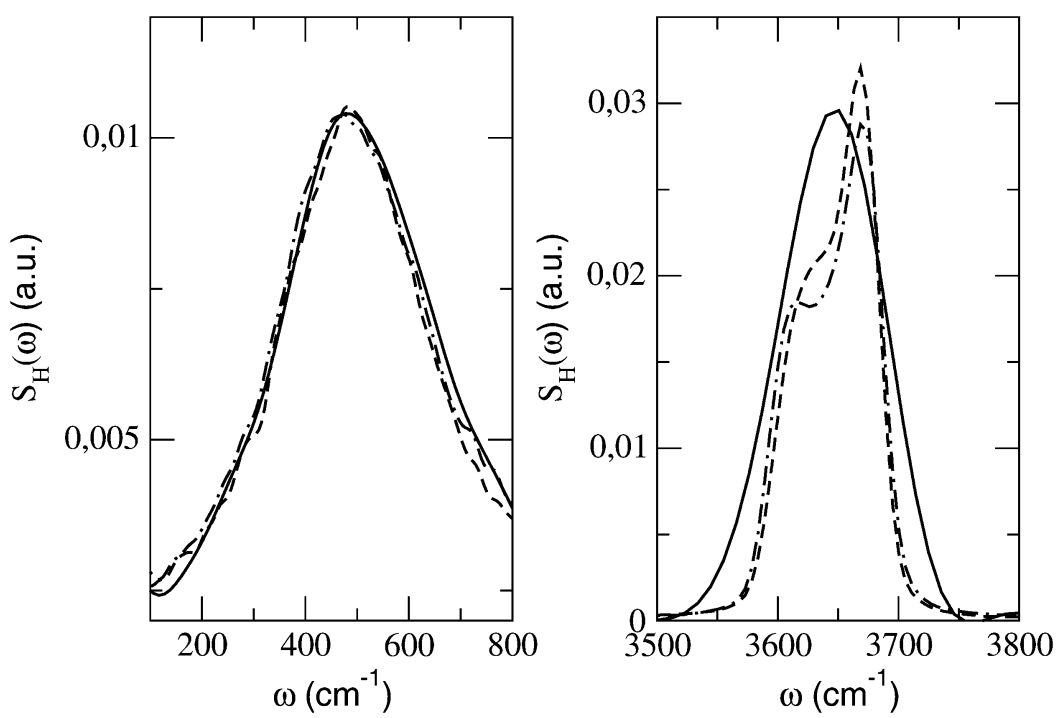

Fig. 3 Spectral densities $S_{H}(\omega)$ of hydrogens for bulk water (full line) and micellar water: water at the interface (dashed line), bulk-like water (dot-dashed line). The two selected regions are: left panel, librational region; right panel, OH-stretching region. 

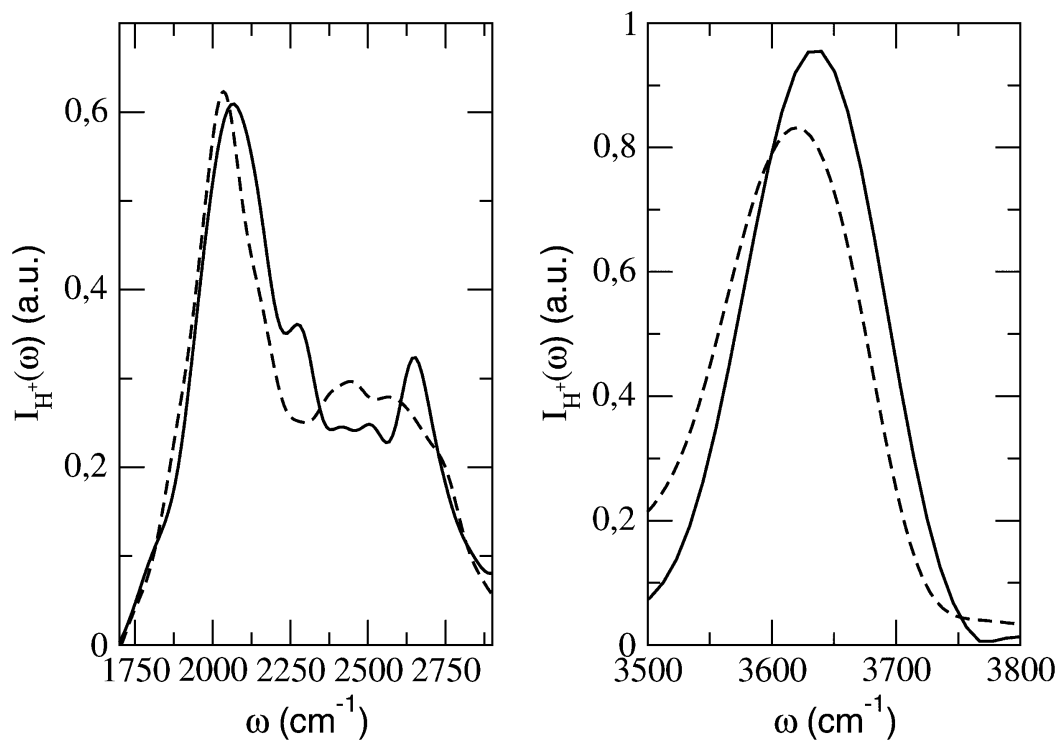

Fig. 4 Proton absorption lineshapes $I(\omega)$ of hydrogens for bulk water (full line) and in the micellar environment (dashed line).

maxima. This band, already observed in bulk unconstrained water at room temperature, ${ }^{17,41}$ remains in the absorption lineshape of protons inside the nanodroplet. The main features of this band are two maxima located in the regions of 1900-2200 and 2300-2700 $\mathrm{cm}^{-1}$. In the case of unconstrained aqueous protons we obtain similar results to those by Voth et $a l .{ }^{17}$ with peaks around 2065 and 2650 wavenumbers. In a previous work ${ }^{41}$ we could associate such bands basically to the contribution from Zundel and Eigen-like structures, respectively. As a justification, we pointed out that, in the framework of the present potential model, band contributions in the 1740-1780 wavenumber interval correspond to the asymmetric bending mode of a Zundel dimer, whereas the collective $\mathrm{OH}$-stretch at $2660 \mathrm{~cm}^{-1}$ corresponds to the stretch of an Eigen complex, both computed in vacuo. The characteristics of the spectrum in micelles, would indicate the existence of both contributions from the two basic structures (Zundel dimer, Eigen cation) to the vibrations of excess protons inside the water pool. Within these environments, we observe the two maxima located around 2035 and $2520 \mathrm{~cm}^{-1}$, i.e. the lower frequency peak presents a moderate red-shift of about $\Delta \omega \sim-30 \mathrm{~cm}^{-1}$ whereas the high frequency band is red-shifted about $\Delta \omega \sim-130 \mathrm{~cm}^{-1}$. We believe that such spectral shifts might be observed in appropriate spectroscopy measurements that, to the best of our knowledge, are not yet available in the literature. Again, the reduction in the spectral bands would correlate with an overall slower dynamics of the molecules lying closer to interfaces.

\subsection{Low frequency spectroscopy: pure water}

Insightful information about the low-frequency part of the water spectrum $\left(0-300 \mathrm{~cm}^{-1}\right)$ can be obtained from the spectral density of oxygen atoms $S_{O}(\omega)$, which can be computed in the same fashion as $S_{H}(\omega)$. Results for $S_{O}(\omega)$ for water inside the micelle are compared with those from bulk unconstrained water in Fig. 5. In bulk water, two main

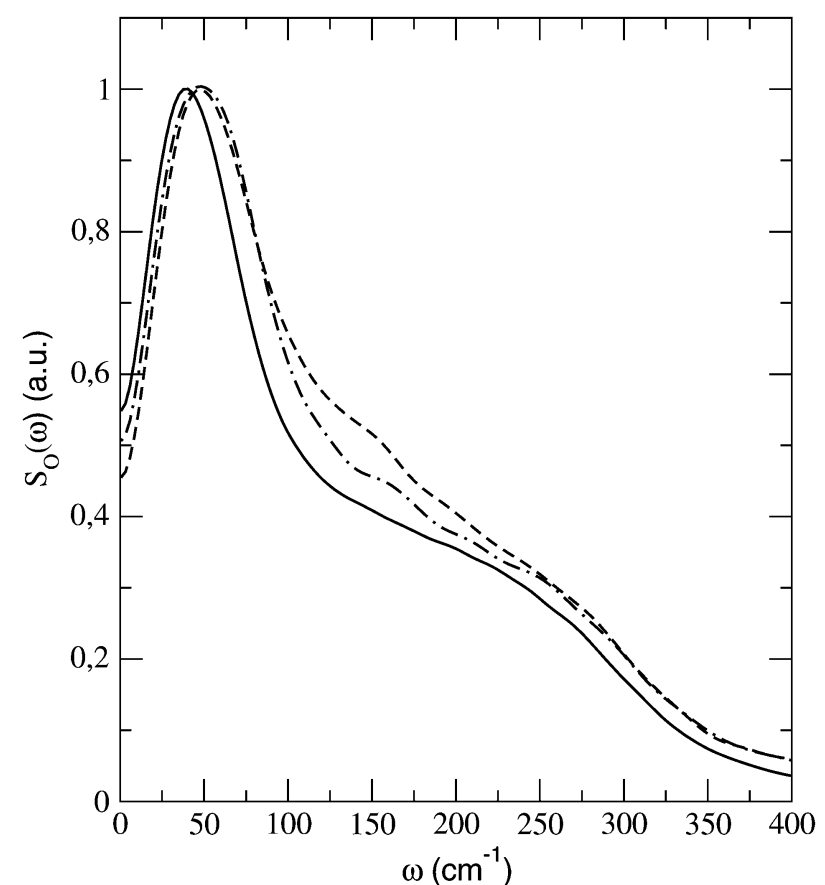

Fig. 5 Spectral densities of oxygens $S_{O}(\omega)$ for bulk water (full line) and micellar water: water at the interface (dashed line), bulk-like water (dot-dashed line).

features are observed: a band around $50 \mathrm{~cm}^{-1}$, related to restricted translational vibrations of a tagged water molecule in the cage of its nearest neighbors; ${ }^{42-44}$ in addition, a shoulder centered around $200 \mathrm{~cm}^{-1}$ appears, which can be attributed to the so-called $\mathrm{O}-\mathrm{H}-\mathrm{O}$ hydrogen-bond stretching vibration. ${ }^{42-44}$ In the case of the lowest frequency peak, a blue-shift of $\Delta \omega \sim 15 \mathrm{~cm}^{-1}$ is observed, for both bulk-like and interfacial water. Here the fact that the micellar pool is so small $\left(w_{0} \sim 2.5\right)$ makes almost no difference between the two groups. In the two cases, the cage effect is slightly milder than in bulk water. 
Concerning the highest frequency feature, we observe a different shape of spectral densities computed in the micellar water pool. In particular, the spectrum for water at the interface has no trace of the shoulder, whereas bulk-like contribution maintains this feature. This observation would indicate the existence of subtle modifications in the hydrogenbond network at the interface, even though most of the water molecules are still four-coordinated, as observed in the detailed hydrogen-bond analysis carried out during our simulations (see ref. 11). However, it should to be kept in mind that such four-fold coordination will be exclusively due to water-water hydrogen-bonds for water located at the central region of the pool, ${ }^{9}$ whereas water molecules at the micellar interface are generally bonded to two (three) water units and to two (one) surfactant sites. So, stretching vibrations of $\mathrm{O}-\mathrm{H}-\mathrm{O}$ units in pure water would become eventually reduced when water is bound to the surfactant headgroups.

\section{Concluding remarks}

This article reports a MD study of the microscopic dynamics of liquid water constrained in a small size non-ionic $\mathrm{C}_{12} \mathrm{E}_{2}$ micelle in vacuo. We considered two main cases: one corresponding to a pure water sample and a second one for a single proton species dissolved in water. The simulation of the excess proton in water was performed through an empirical valence bond methodology. Our results shed light on properties such as molecular reorientations, translational diffusion and molecular spectroscopy.

The results obtained for both self-diffusion coefficients and reorientational times suggest the slowing down of water dynamics inside the microemulsion: translational self-diffusion coefficients are roughly one half the corresponding values in unconstrained bulk water, whereas reorientational times are markedly longer than in bulk water. In order to have a deeper look at such features, a classification of water molecules was considered: interfacial water for molecules whose center of mass is less than $3 \AA$ apart from any surfactant site, and bulk-like water for the remaining molecules. Calculations of dynamical properties were performed for the two selected groups, leading to longer reorientational times and slightly smaller diffusion coefficients for water at the interface versus bulk-like water. All these results suggest: (i) in such a small micelle, water has qualitatively similar diffusional behavior both in interfaces and in the central region of the pool; (ii) the presence of the surfactants tends to slow down the mobility of water molecules. However, we should keep in mind that ours is a basically hydrophilic micelle and that for ionic micelles these features should be very different. In our calculations, no counter-ion was included. We tend to think that its inclusion would produce significant changes in the configurational energy of the system, whereas much smaller variations in the dynamics of local proton-water environments would be expected. As a possible indication of those changes in dynamics, we should point out that Hasegawa ${ }^{45}$ observed only minor changes in the probe excitation spectra corresponding to a local intramicellar $\mathrm{pH}$ of $\sim 5$, regardless of the adjusted $\mathrm{pH}$ of the bulk solution before solubilization (between 3-11).
Spectroscopic properties have been analyzed for two different systems: pure water and water plus an excess proton. We studied four frequency ranges: (1) the low-frequency region $\left(0-300 \mathrm{~cm}^{-1}\right)$; (2) the librational region $\left(300-800 \mathrm{~cm}^{-1}\right)$; (3) the region between $1800-2800 \mathrm{~cm}^{-1}$, specifically relevant for the excess proton spectrum, and (4) the $\mathrm{OH}$-stretch vibration region $\left(3200-4000 \mathrm{~cm}^{-1}\right)$. After comparison of spectral densities and absorption lineshapes for interfacial and bulk-like water, we obtained spectral shifts and band splitting features in the two higher frequency regions, whereas no significant changes were observed in the librational region. From a general point of view, such frequency shifts can be essentially due to confinement effects and they are somewhat influenced by the presence of the surfactant species. Finally, the differences in the shape of $S_{O}(\omega)$ (which is related to the frequency region between $0-300 \mathrm{~cm}^{-1}$ ) can be explained by the fact that the geometry of the hydrogen-bond network around the micellar interface is qualitatively different from that of bulk-like water.

\section{Acknowledgements}

The authors gratefully acknowledge financial support from the Direcció General de Recerca de la Generalitat de Catalunya (Grant 2005SGR-00779) and the Ministerio de Educación y Ciencia of Spain (Grant FIS2006-12436-C02-01). DL and JR are staff members of CONICET-Argentina.

\section{References}

1 P. K. Luisi and B. E. Straub, Reverse Micelles: Biological and Technological Relevance of Amphiphilic Structures in Apolar Media, Plenum Press, New York, 1984.

2 Structure and Reactivity in Reverse Micelles, ed. M. P. Pileni, Elsevier, Amsterdam, 1989.

3 M. N. Jones and D. Chapman, Micelles, Monolayers, and Biomembranes, John Wiley, New York, 1995.

4 N. Nandi, K. Bhattacharyya and B. Bagchi, Chem. Rev., 2000, 100, 2013.

5 N. E. Levinger, Science, 2002, 298, 1722.

6 I. R. Piletic, D. E. Moilanen, D. B. Spry, N. E. Levinger and M. D. Fayer, J. Phys. Chem. A, 2006, 110, 4985.

7 A. M. Dokter, S. Woutersen and H. J. Bakker, J. Chem. Phys., 2007, 126, 124507.

8 D. E. Moilanen, N. E. Levinger, D. B. Spry and M. D. Fayer, J. Am. Chem. Soc., 2007, 129, 14311.

9 J. Rodríguez, J. Martí, E. Guàrdia and D. Laria, J. Phys. Chem. B, 2007, 111, 4432.

10 R. Allen, S. Bandyopadhyay and M. L. Klein, Langmuir, 2000, 16, 10547.

11 Among the population of interfacial water, about $24 \%$ has been found to form double hydrogen-bonds with acceptor $\mathrm{O}-\mathrm{O}$ sites of a given surfactant.

12 S. Senapati and M. L. Berkowitz, J. Chem. Phys., 2003, 118, 1937.

13 J. Lobaugh and G. A. Voth, J. Chem. Phys., 1996, 104, 2056.

14 U. W. Schmitt and G. A. Voth, J. Phys. Chem. B, 1998, 102, 5547.

15 U. W. Schmitt and G. A. Voth, J. Chem. Phys., 1999, 111, 9361.

16 T. J. Day, U. W. Schmitt and G. A. Voth, J. Am. Chem. Soc., 2000, 122, 12027

17 T. J. Day, A. V. Soudackov, M. Cuma, U. W. Schmitt and G. A. Voth, J. Chem. Phys., 2002, 117, 5839.

18 H. J. C. Berendsen, D. van der Spoel and R. van Drunen, Comp. Phys. Comm., 1995, 91, 43.

19 E. Lindahl, B. Hess and D. van der Spoel, J. Mol. Mod., 2001, 7, 306.

20 A. W. Schuettelkopf and D. M. F. van Aalten, Acta Crystallographica, 2004, D60, 1355. 
21 P. A. Madden and D. Kivelson, Adv. Chem. Phys., 1984, 56, 467.

22 D. Lankhorst, J. Schriever and J. C. Leyte, Ber. Bunsenges. Phys. Chem., 1982, 86, 215.

23 M. Praprotnik and D. Janežič, J. Chem. Phys., 2005, 122, 174103.

24 I. R. Piletic, D. E. Moilanen, N. E. Levinger and M. D. Fayer, J. Am. Chem. Soc., 2004, 128, 10366.

25 J. Faeder and B. Ladanyi, J. Phys. Chem. B, 2000, 104, 1033.

26 M. Wong, J. K. Thomas and M. Graetzel, J. Am. Chem. Soc., $1976,98,2391$.

27 M. Wong, J. K. Thomas and T. Nowak, J. Am. Chem. Soc., 1977, 99, 4730 .

28 P. E. Zinsli, J. Phys. Chem., 1979, 83, 3223.

29 G. Carlstroem and B. Halle, Langmuir, 1988, 4, 1346.

30 S. A. Corcelli and J. I. Skinner, J. Phys. Chem. A, 2005, 109, 6154

31 D. A. McQuarrie, Statistical Mechanics, Harper and Row, New York, 1976.

32 R. Vuilleumier and D. Borgis, J. Chem. Phys., 1999, 111, 4251.

33 J. Martí, E. Guàrdia and J. A. Padró, J. Chem. Phys., 1994, 101, 10883.

34 J. Martí, J. A. Padró and E. Guàrdia, J. Chem. Phys., 1996, 105, 639
35 J. Martí, G. Nagy, E. Guàrdia and M. C. Gordillo, J. Phys. Chem. $B, 2006,110,23987$.

36 A. I. Kolesnikov, J.-M. Zanotti, C.-K. Loong, P. Thiyagarajan, A. P. Moravsky, R. O. Loufty and C. J. Burnham, Phys. Rev. Lett., 2004, 93, 035503.

37 J. Martí and M. C. Gordillo, Phys. Rev. B, 2001, 63, 165430.

38 S. H. Chen, K. Toukan, C. K. Loong, D. L. Price and J. Teixeira, Phys. Rev. Lett., 1984, 53, 1360.

39 A. M. Dokter, S. Woutersen and H. J. Bakker, Proc. Natl. Acad. Sci., 2006, 103, 15355.

40 A. M. Dokter, S. Woutersen and H. J. Bakker, Phys. Rev. Lett., 2005, 94, 178301 .

41 D. Laria, J. Martí and E. Guàrdia, J. Am. Chem. Soc., 2004, 126, 2125 .

42 J. A. Padró and J. Martí, J. Chem. Phys., 2003, 118, 452.

43 A. De Santis, A. Ercoli and D. Rocca, J. Chem. Phys., 2004, 120 1657.

44 J. A. Padró and J. Martí, J. Chem. Phys., 2004, 120, 1659.

45 M. Hasegawa, Langmuir, 2001, 17, 1426. 